\title{
FINITE GROUPS.
}

Theory and Applications of Finite Groups. By G. A. Millen, H. F. Blichfeldt, and L. E. Dickson. New York, Wiley, 1916. 8vo. $17+390$ pp.

DURING the last half-century the theory of groups has been explored with great vigor and success, and it is now an imposing body of doctrine. Its importance is principally due to the fact that it enters into the very warp and woof of a large number of mathematical textures. "Many branches of mathematics are nothing but the theory of invariants of special groups." This is especially true of geometry, as was made clear once for all by Klein. Geometry is not one science, but an infinite variety of sciences, depending on the choice of the group on which it is based. As the fundamental group becomes larger and more extensive, the corresponding geometry becomes less extensive and more fundamental. Thus, beginning with the elementary geometry of congruent figures, we obtain in succession a number of different geometries among which are the geometry of similar figures, affine geometry, projective geometry, conformal geometry, and analysis situs.

From a purely abstract standpoint groups may be classified in various ways: they are either abelian or non-abelian, finite or infinite, continuous or discontinuous. The principal classes of concrete groups are permutation groups, linear groups, and groups of non-linear transformations, together with numerous geometrical and mechanical applications. Even in the study of point sets and other aggregates groups have been found useful; for instance, in Bernstein's proof that if two cardinal numbers $n \alpha$ and $n \beta$ are equal, then $\alpha=\beta$.

In a linear group the transformations may be homogeneous or fractional, singular or non-singular, but the most striking principle of classification is that which depends on the nature of the coefficients, which may belong to an infinite field, a finite field, or no field whatever. If the coefficients belong to an infinite field or domain, for instance that of all complex numbers, we obtain a most important class of groups, both continuous and discontinuous, with which are closely asso- 
ciated groups of collineations and other geometric transformations. If the coefficients belong to a finite (or Galois) field, of which a special case is that in which they are residues with respect to a prime modulus, the groups are themselves necessarily finite. But the coefficients may not belong to any field. Thus they may be residues with respect to a composite modulus, or with respect to a number of different moduli, or with respect to a modular system. Very little has been done toward the investigation of this class of groups.

When we come to groups of non-linear and in particular of non-algebraic transformations, the possible developments are too vast to contemplate and for the most part lie buried in the future. In the case of birational transformations a beginning has been made. The tremendous variety of geometric groups is evident when we recall that the geometry under consideration may be non-euclidean as well as euclidean, that its number of dimensions may be $n$ or even infinite, that the elements which are transformed may be not merely points, but lines, circles, spheres, etc., and finally that the set of such elements, say of points, instead of forming a continuum, may form a point set of the most general type. Since many physical and chemical changes also form groups, Lie was almost justified in his enthusiastic query, "What are the phenomena of the physical and material universe but the transformations of an enormous group, of which the laws of nature are the invariants?"

Even that part of the theory which has arrived at a fair degree of completeness and finish is far too extensive for a single treatise. The volume before us is restricted to finite groups and indeed to certain selected topics in their theory and applications; linear congruence groups are barely touched upon. The book is written by three recognized authorities on various phases of the subject. Considerable original material is included by each of the authors either in the form of new developments or of simplifications in the proofs of the older theorems. The work is strictly up to date; it contains some of the very latest results on the topics treated.

G. A. Miller, who is responsible for Part I, on abstract and permutation groups, is thoroughly at home in this field; indeed, he has contributed so extensively to its theory and has created so many new and valuable phases of it that he may fairly be called one of the world's leading authorities on 
the subject. Part II, by Blichfeldt, is devoted to finite groups of linear homogeneous transformations whose coefficients belong to the domain of complex numbers. The writer is eminently fitted for this task, because he is himself responsible for a new and brilliant method of attack, which enabled him to arrive at the first exhaustive enumeration and discussion of the groups in four variables. Part III, by Dickson, covers the Galois theory of equations and applications to certain geometric problems. In this case also the writer is thoroughly qualified for his task. For he is a very productive mathematician in this and various other fields, who has not only given a definitive and apparently final form to the theory of linear congruence groups and thereby added tremendously to our knowledge of simple groups, but has also contributed largely to the progress of mathematics in many other directions.

Naturally the result of this kind of collaboration is a volume consisting practically of three separate treatises, and yet a certain amount of unity has been attained; while Parts II and III are largely independent of one another, they both presuppose a knowledge of some of the more elementary sections of Part I. We already possess treatises and textbooks on finite groups by Jordan and de Séguier in French, by Bianchi in Italian, by Klein, Weber, and Netto in German, and by Burnside, Hilton, and Dickson in English. But this new volume will easily bear comparison with any of them, not only in its wealth of valuable material, much of which is new, but in vigor and directness of approach, in elegance of treatment, and in the thoroughly interesting way in which many of the results are presented. Certain flaws, mostly of a secondary nature, will presently be pointed out, but every reader must admit the general excellence of the work. There is a graceful and becoming dedication to Camille Jordan, " whose fundamental investigations in the theory and applications of finite groups enriched the subject to the extent of converting it into a fundamental branch of mathematics and furnished in a large measure the inspiration for the subsequent great activity in this field."

In Part I, which covers about half the book, the systematic exposition is preceded, in Chapter 1, by a few simple examples of concrete groups, including the groups of rotations of the equilateral triangle and of the square, a trigonometric illustration of the octic group, and a few simple congruence groups 
and groups of matrices. In this way the wind is very properly tempered to the shorn lamb, namely, the beginner. Another admirable feature, pedagogically, is the fact that substitution (or permutation) groups are studied before abstract groups. Indeed, one has the feeling, in reading Part I, that every abstract group is clothed in the flesh and blood of a substitution group, and in particular of a regular group.

In Chapter 2 we meet with substitution groups and their properties, including transforms, co-sets, Sylow's theorem, transitive and intransitive groups, primitive and imprimitive groups, the simplicity of the alternating group, the class of a group, the holomorph of a group, and a determination of all the groups of degree $\leqq 5$. Chapter 3 is devoted to the more fundamental theorems on abstract groups, including the cyclic, dihedral, and dicyclic groups, commutator subgroups, $\phi$-subgroups, inner isomorphisms, and the representation of a group as a transitive or regular substitution group. In Chapter 4 we come to abelian groups, their invariants, characteristic subgroups, and groups of isomorphisms; also the roots of their elements and the number of elements of a given order; abelian groups which are conformal with non-abelian groups, non-abelian groups in which every subgroup is abelian, and Hamilton groups, in which every subgroup is invariant. The bitter pill of pure abstraction is sugar-coated for us with the delectable group of totitives. Chapter 5 is concerned with prime-power groups, their subgroups of various types, their groups of isomorphisms, and finally a method of constructing all the possible groups of order $p^{m}$. A proof is given of the important new theorem that the number of elements in a set of independent generators of a prime-power group is an invariant of the group. In Chapters 6 and 7 the results are more largely due to Miller's own research than in the other chapters, and are of extremely great value and interest. Chapter 6 is devoted to groups having simple abstract definitions and includes the groups generated by two elements having a common square, the groups of the regular polyhedrons, and various generalizations of the latter. Chapter 7 contains an intensive study of groups of isomorphisms. Chapter 8 is concerned with series of composition, chief series, solvable groups, and unsolvable groups of low composite orders.

Now for a few adverse criticisms. The author of Part I does not always get in touch with the beginner; some of the 
initial difficulties of the subject are slurred over. For instance, on page 16 , in the third sentence, the adverb "evidently" might easily be regarded as ironical by the beginner. Moreover, the fourth sentence on the same page is not true, unless the cycles are assumed to have no letters in common and therefore to be different from the cycles in the product written down in the next paragraph. A number of terms and phrases are not carefully or explicitly defined; e. g., " transitive constituent groups " on page 33 , line 25 , “ $(1, m)$ correspondence" on page 39 , line 1 , the "holomorph" and "group of isomorphisms" of an abstract group. The fact that a simple isomorphism is a $(1,1)$ correspondence in which the products of corresponding elements are themselves corresponding elements is nowhere explicitly mentioned; and this is all the more misleading since the term $(1,1)$ correspondence is used as synonymous with simple isomorphism. In the middle paragraph of page 103 it is implied that a subgroup can be generated by operators not belonging to the subgroup. On pages 34 and 37 no explanation is given as to what is meant by " regarding several substitutions as one substitution."

The following erroneous or ambiguous statements have been noted. On page 70, lines 9,10 , the elements of the commutator are not to be permuted in every possible manner, as there stated, but only in such a way that it may remain a commutator. On page 34, line 3 from the bottom, "the symmetric group" should read "two symmetric groups." On page 53 , line 3 from the bottom, "of the form $l+m k$ ", should read " $l+m_{1} k$ and $l+m_{2} k . "$ In $\S 38$, second paragraph, first sentence, the adjective "low" should be inserted before " order," in order to avoid an apparent conflict with page 89 , lines 20-26. On page 99, first theorem, last line, "equal to or greater than " should read "divisible by." On page 179, line 5, “ $\alpha$ conjugates of $H_{1}$ " should read " $\alpha-1$ terms." On page 185, lines 12, 13, "whose order exceeds unity" should read "which is non-invariant." On page 113, last line, the article "the" should be omitted, as it implies that there is only one Sylow subgroup of each given order. The first sentence on page 174 contains the erroneous statement that the subgroups there mentioned are invariant; but the error is corrected by implication in the next paragraph.

Part II, consisting of Chapters 9-13, gives us a more direct approach to the finite groups of linear transformations, 
especially those in three variables, than is contained in any earlier text. We start off, in Chapter 9, along a well-beaten track, which takes us to the characteristic equation, Hermitian invariants, unitary transformations, reducible or intransitive groups, the canonical form, etc. In Chapter 10 we encounter the groups in two variables, which are determined by Klein's original geometric method, somewhat simplified. Thus we get a second view of the groups of the regular polyhedrons. Chapters 11 and 12 contain the author's own powerful method of handling the groups in three variables. As compared with the older methods of Jordan, Valentiner, and others, it is remarkable for its brevity and completeness. An important rôle is played by Kronecker's theorem on roots of unity, and also by the theorem that no prime $>7$ can divide the order of a primitive group in three variables. In Chapter 13 we have a brief introduction to the theory of group characteristics and one or two of its applications.

The last formula on page 230 would be somewhat clearer if it were stated that $\alpha_{i}$ is a multiplier of $T_{i}$ and that the notation $\alpha_{i}\left(y_{1}, y_{2}\right)$ means $\left(\alpha_{i} y_{1}, \alpha_{i} y_{2}\right)$. A misleading reference to "regular polygons" is made on page 219 , line 14 ; for of course in the case of the dihedral groups the polygons are not regular. An unusual and rather unfortunate nomenclature is employed in $\$ 77$, line 1 , and also in Part III, on page 373 , line 7 . While a set of values of the unknowns satisfying a system of equations, one for each unknown, is commonly called a solution of the system, the authors call it a set of solutions, the value of each unknown being a solution. In the definition on page 194 the condition that the equations " can be solved" for the new variables is hardly drastic enough; what is meant is that the equations must possess exactly one solution (or set of solutions, to use the author's language).

Part III, consisting of Chapters 14-20, begins with the Galois theory of equations, the treatment of which is somewhat similar to that of the author's excellent "Theory of Algebraic Equations," except that it is simpler and briefer, and that the comparatively easy case in which the domain of rationality involves only constants is wisely given first. Chapters 15 and 16, on the necessary and sufficient conditions for solvability by radicals, including a discussion of the cyclotomic equation and of the general cubic and quartic, naturally connect up with the chapter on solvable groups in Part I. 
We now come to some interesting and valuable applications which show what a powerful tool the theory of groups is in solving many problems in geometry. In Chapter 17 we have constructions with ruler and compasses, in Chapter 18 the nine inflection points of a plane cubic curve, and in Chapter 19 the twenty-seven straight lines on a cubic surface as determined by means of their double-six configurations and the twenty-eight bitangents to a quartic curve as determined by means of their Steiner sets and Aronhold sets. The intimate connection between the last two problems is well shown. For the bitangents there is given an exhibition of Hesse's and Cayley's unsymmetric notation and also a purely algebraic derivation of the symmetric notation which was originally derived from the properties of theta functions. The methods of proof are largely the author's own and as compared with earlier treatments like that in Weber's Algebra, for instance, they show a vast improvement. Especially noteworthy is the determination of the number of real elements in each case.

The necessary geometric theorems are not presupposed, but are derived in the text; so that the discussion is intelligible to a reader with no knowledge of homogeneous coordinates or projective geometry; or at least it would be intelligible to him if some explanation had been given of the so-called points and lines at infinity, and if the cases in which these ideal elements are included had been carefully distinguished from those in which they are not. This has not been done; hence a certain amount of confusion. E. g., in $\$ 176$, fourth sentence, we are supposed to pass from a triangle of reference to Cartesian coordinates by setting $x_{2} / x_{1}=x, x_{3} / x_{1}=y$. But this special kind of triangle of reference has not been defined in the text. Moreover, in the definition of the ordinary triangle of reference, on page 327 , the condition $\Delta \neq 0$ is not sufficient, for it would allow two sides of the triangle to be parallel. Again, on page 346, line 1, the surface may be a hyperbolic paraboloid as well as a hyperboloid of one sheet, even when the lines $c_{2}, c_{3}, c_{4}$ are all real. In the first paragraph of $\S 187$, in order to avoid infinite values of $m$ or $\xi$, the $x$-axis would have to be chosen so as to be neither parallel nor perpendicular to any bitangent.

The following typographical errors have been noticed.

Page 24, second footnote, second line. For "her" read "here."

Page 48, line 26. For " $s_{1}$ " read " $s_{2}$." 
Page 68, line 21. For "congredient" read "cogredient."

Page 140, line 9. Interchange "order" and "product."

Page 163, line 24. For "operator" read "operators."

Page 183, line 24. For "subgroups" read "subgroup."

$\S 91$, line 2. For the second " $w$ " read " $w$."

Page 212, exercise 1. After "transformation" insert "of determinant unity."

Page 345, footnote, line 5. For " $c_{3}$ " read " $c_{4}$."

Page 381, line 6 from bottom. For "tenary" read "ternary."

In spite of a few slight blemishes, the book is an extremely valuable contribution to mathematical literature, one that for many years to come will be indispensable to the worker in the domain of finite groups.

Arthur Ranum.

\section{SHORTER NOTICES.}

L'Annuaire du Bureau des Longitudes pour l'An 191\%. Paris, Gauthier-Villars.

AN interesting feature of the Annuaire for 1917 is the extensive "Notice"- "L'Avance de l'heure légale pendant l'été de l'année 1916," by M. J. Renaud. In its ninety-odd pages he traces the history of daylight-saving from the initiation of the mean time of Paris as the legal hour for France and Algeria to the passing of the law last year which put the clocks forward one hour during the summer months. A resumé of the opinions of scientific bodies, of the press, of the Chamber of Commerce of Paris, and even of the Académie des Sports on the last change is followed by reports of the discussions which took place in the Senate and Chamber of Deputies; there is much material here for those who are interested in the possible adoption of a similar change on this continent. A further section is devoted to the various measures which were taken to avoid confusion in making the change of hour in the spring and fall, and still another section to what has been done in other countries. M. Renaud concludes finally that concerted measures by international agreement are necessary if proper stability in the indication of legal time is to be effected.

In the other "Notices" M. G. Bigourdan gives an account of the Babylonian calendar in some detail, M. M. Hamy 